\title{
A Cross-sectional Comparative Study of Movement Distances and Speed of the Players and a Ball in Basketball Game
}

\author{
Watartu Oba* and Tomoyasu Okuda** \\ *Faculty of Humanities, Okinawa University (Faculty of Education, Osaka Kyoiku University) \\ 555 Kokuba, Naha, Okinawa 902-8521 Japan \\ owataru1110@gmail.com \\ **Department of Sport Education, Hokkaido University of Education \\ 2-34 Midorigaoka, Iwamizawa-City, Hokkaido 068-8642 Japan \\ [Received May 20, 2008; Accepted December 11, 2008; Published online April 20, 2009]
}

\begin{abstract}
The purposes of this study were: 1) to measure the movement distances and the movement speeds of each player or the ball in a regular basketball game: and 2) to propose effective coaching material about players' physical conditioning through the cross-sectional comparative method of that game performance. Three basketball games were analyzed. They were the xxth All-Japan Inter-High School Basketball Championship women's semifinal (A-high school vs. Bhigh school in which A-high school won, 74-68), the xxth Western Collegiate Basketball Championship women's consolation match to decide third place (C-college vs. D-college in which $C$ college won, $75-68$ ), and the $200 x-x+1$ W-League Playoff Final 1st game (E-team vs. Fteam in which E-team won, 98-73). In this study, the movement distances/speeds of all the players and the ball were calculated by 3-dimensional photography analysis in which the DLT method was used. Main results were as follows. 1) The average and standard deviation (S.D.) of the movement distance of all players were: high school players, $5587 \pm 171 \mathrm{~m}$; college players, $5576 \pm 202 \mathrm{~m}$; and WJBL players, $6177 \pm 264 \mathrm{~m}$. Moreover, the average and standard deviation of the movement distance per 1 minute were: high school players, 93.4 $\pm 3.1 \mathrm{~m}$; college players, $100.4 \pm 4.1 \mathrm{~m}$; and WJBL players, $94.2 \pm 3.5 \mathrm{~m} .2$ ) The average and S.D. of the fastest movement speed were: high school players, $7.03 \pm 0.51 \mathrm{~m} / \mathrm{s}$; college players, $7.41 \pm 0.26 \mathrm{~m} / \mathrm{s} ; \mathrm{WJBL}$ players, $8.01 \pm 0.46 \mathrm{~m} / \mathrm{s}$. 3) From calculation of the frequency distribution for the speed ratio to the fastest movement speed, the ratio of the movement intensity in a game was high : middle:low $=1: 4: 5$. These finding revealed although the quantity of motion and movement intensity in the game did not differ much by age, each player's individual capability differed clearly.
\end{abstract}

Keywords: game performance analysis, basketball, movement distance, movement speed, cross-sectional method study

[International Journal of Sport and Health Science Vol.6, 203-212, 2008]

\section{Introduction}

In ball games typified by basketball, roles for tactics to win a game are very important (Uchiyama, 2007), and game performance analysis methods have been used actively for analysis of behavior in such collective tactics (Oba \& Okuda, 2007). By quantifying various events occurring in sports, they have been used for the purposes of training and technical practice in the coaching process and team management, feedback to planned tactics, and improvement in skills performance of individual players and their team (Ohashi, 1999).

Generally, game performance analysis methods may be classified into those of game analysis and game statistics (Suzuki \& Nishijima, 2002). Game analysis qualitatively evaluates team performances, e.g., offense, defense, and organization, through visual confirmation of coaches and experts. Although superficial events in competitive sports are nonrecurrent and individual, they are based on deep, 
universal structures and principles (Uchiyama, 2004). By using game analysis methods, games can be evaluated comprehensively from the analyst's experience and universal principles may be clarified, but these methods still have important issues of low reproducibility and the need for extensive generalization to provide a common understanding among coaches because the evaluation involves the analyst's subjectivity and arbitrariness. On the other hand, game statistics evaluates game performance of individual players and their team by using enumerated data such as team points scored, opponent points scored, number of shots attempted, and percentage of shots made. The evaluation is fed back to actual coaching fields as short-term information utilized for tactics change and player substitution in the game etc., medium-term information utilized for post-game planning of tactics for the next game etc., and longterm information utilized for practices, training, and player selection for enhancement of players and their team etc (Ohashi, 1999). However, it has pointed that coaches in the field desire game performance analysis which provides medium-term information like scouting of the opponent for the next game and long-term information for task setting in practices and training of players on their team, rather than short-term information (Kato, 1999).

Game performance analysis is acknowledged as important and necessary in basketball as in other ball games, and has been researched in many studies. Okuda et al. (2005) classified previous research on game performance analysis into quantitative research studies and qualitative research studies based on the method used and subjects analyzed, and then they reviewed both types. Quantitative research is aimed at evaluating competitive capabilities of players and teams and/or comprehending winning or losing of games in terms of a quantity obtained by measuring and analyzing enumerated data quantitatively; it is typified by research that is intended to indicate analytical results of games in real time by taking advantage of the processing capacity of computers (Kodama et al., 1996), research that determines factors for winning or losing by analyzing games in time sequence with a concept called Droughts (Ishimura et al., 1988), and research that evaluates the entire competitive capabilities of individuals and groups by processing their enumerated data statistically (Oga et al., 2001). On the other hand, qualitative research aims at evaluating competitive capabilities of players and teams in terms of quality by analyzing positional information and movement patterns of players and patterns of team tactics qualitatively and determining value and significance of plays under a game situation; it is typified by research that records tactics of a game from playing back video tapes of game recordings (Uchiyama et al., 2001), research that analyzes coordinated plays in time sequence by a graphic method (Iwamoto et al., 2001), and research that analyzes the positional relationship between two players by using a 2-dimensional photography analysis method such as direct linear transformation (DLT) (Kato et al., 1993). Of published research studies using game performance analysis, $82 \%$ have been identified as quantitative research and only $18 \%$ were qualitative research, based on the above characterizations (Okuda et al., 2005). A reason for this may be that data in quantitative research are easy for analysts to objectively evaluate because they are easy to collect or often enumerated. However, Uchiyama (2004) and Suzuki (2005) pointed out: although data in quantitative research, as long as they are enumerated, can be analyzed for performances per game, it is impossible to analyze performances per play or comprehend what roles individual players fulfill in relation to others and their team during a game because performance exerted during the game can only be comprehend in a onesided way and during the final phase. Thus, information derived from quantitative research, which has not been evaluated directly and qualitatively, may be insufficient for application as medium- or long-term information which is fed back to coaches in the field (Kato, 1999). Also, qualitative research still has some issues. Many studies assume only limited phases and situations and further, calculated data are not obtained directly and objectively and have not been examined for reliability and validity from the viewpoint of deviation from actual data. Furthermore, the recorded data can involve the analyst's subjectivity and arbitrariness because they were evaluated by his or her subjective judgment.

Consequently, to feed back medium- and long-term information to coaches in the field, it is important to analyze performance of players appearing in actual games using objective indices which do not involve the analyst's subjectivity and arbitrariness. In order to resolve that issue, currently, new research on game performance analysis that directly measures movement distance or other parameters of players during a game by using a triangular surveying method or a photography analysis method to measure positional 
information of the players is emerging (Ohashi, 1999; Kan et al., 2000). Of these research studies, game performance analysis for players' movements using 3dimensional photography analysis methods requires a long time for analysis because all players' movements are recorded concurrently, although they have an advantage that the position of all players and the game ball can be computed as time sequential data.

In basketball, there have been a few studies that analyzed game performance photographically with the DLT method: Iwamoto et al. (1998) analyzed the speeds and distances of offense and defense players during the cut-in play focusing on their one-on-one movements; Kato et al. (1993) analyzed the positional relationship between passer and shooter; and Matsumoto et al. (1998) obtained the movement distances and average speeds of only three players in one team during a practice game. These studies could exclude the analysts' subjectivity and arbitrariness to demonstrate correct positional information of players; however, they did not analyze the collective tactics and game performance on actual courts because they were targeting two-on-two collective tactics or analyzing practice situations which involved experimental factors.

On the other hand, Oba and Okuda (2007) and Okuda et al. (2007), who targeted an official game, measured correct positional information for players and the ball as data of 3-dimensional positional coordinates in time sequence and used these data to compute data on physical fitness factors (e.g., movement distances and speeds of the players and the ball during the game) and to compute data on tactical factors (e.g., relationships among the players, spatial quantifications using a pressure distribution model). As a result, they succeeded in objectively analyzing the movements of the players and the ball during the game and further they objectively evaluated and qualitatively analyzed the team tactics. However, because they used only one game, it was desirable to expand their results to applications in coaching and to broaden the targets to be analyzed.

Thus, the present study targeted three official basketball games, one each from a high school, a college, and the Women's Japan Basketball League (WJBL). The purposes were to demonstrate movement distances and speeds of players at these playing levels by measuring the movements of the players and the ball during each game with a 3-dimensional photography analysis by the DLT method, and to obtain effective knowledge about training instructions on previously- unrevealed physical fitness factors during basketball games through that cross-sectional analysis.

\section{Methods}

\subsection{Sample}

For analysis of a high school basketball game, the xxth All-Japan Inter-High School Basketball Championship women's semifinal in $200 x+2$ was used in which A-high school played against B-high school (A won 74-68). For analysis of a college basketball game, the xxth Western Collegiate Basketball Championship women's consolation match to decide third place in $200 \mathrm{x}$ was used in which C-university played against D-university, (C won 75-68). For analysis of a WJBL game, the $200 x-x+1$ W-League Playoff Final 1st game was used in which E-team played against F-team (E won 98-73). In all games, the full length of the game was analyzed excluding the halftime, intervals between periods, and timeouts. Video recordings were done with permission of the Championships Committee of the xxth All-Japan High School Basketball Championships, the Western InterCollegeate Basketball Federation, and the Osaka Basketball Association.

\subsection{Data collection}

Positional information of players and the ball during the games was recorded with the DLT method in which 2-dimensional images recorded by plural video cameras were converted to 3-dimensional coordinates. Two to four digital video cameras synchronized by an LED synchronizer (PTS-110; DKH Co., Ltd., Tokyo, Japan) were placed at a shooting angle of 70-110 degrees from a stand in the gymnasium, and the full length of the game was recorded at $30 \mathrm{~Hz}$. For video recording, wide conversion lenses, which can distort images, were not used, and each camera was placed so as to bring identical reference points (e.g., intersections of the sideline and endline, the hoops) in view.

After the frames recorded by the digital video cameras were digitalized every $1 / 4$ seconds via a video capture board and acquired on a computer, the coordinate data $(\mathrm{x}, \mathrm{y}, \mathrm{z})$ of all players on both teams $(n=10)$ and the ball were determined by using FrameDIAS II 3-dimensional analysis software (DKH Co., Ltd., Tokyo, Japan). For this digitalization, the lumbar part of the players was defined as their position as done 
by Oba and Okuda (2007). For the 3-dimensional coordinates, the $\mathrm{X}$ axis was parallel to the sidelines, the $\mathrm{Y}$ axis was parallel to the endlines, and the $\mathrm{Z}$ axis was set in the vertical direction. Over 5 meter tall poles for calibration of digitalization were set up on the 4 intersections of the sideline and endline and were used as coordinate points. The standard errors of these calibration points between the measured values and the post-restructuring estimated value for the $\mathrm{X}, \mathrm{Y}, \mathrm{Z}$ axes were $0.01 \mathrm{~m}, 0.01 \mathrm{~m}$, and $0.02 \mathrm{~m}$ in the high school game; $0.02 \mathrm{~m}, 0.01 \mathrm{~m}$, and $0.01 \mathrm{~m}$ in the college game; and $0.03 \mathrm{~m}, 0.01 \mathrm{~m}$, and $0.04 \mathrm{~m}$ in the WJBL game, respectively.

\subsection{Data analysis}

Through the numerical process of the 3-dimensional coordinates that were computed by the above-described analysis method, the movement distances and the movement speeds of the 10 players on the court and the ball were determined. The playing position of the bench players was treated as the same as that of their corresponding starters.

\section{Results}

\subsection{Movement distances of players and ball}

Tables 1, 2, and 3 show movement distances of the players and the ball in the high school, college, and WJBL games, respectively. The mean and standard deviation (S.D.) of movement distance of the players per game were $5587 \pm 171 \mathrm{~m}$ for the high school players, $5576 \pm 202 \mathrm{~m}$ for the college players, and $6177 \pm 264 \mathrm{~m}$ for the WJBL players; those per period were $1397 \pm 78 \mathrm{~m}$ for the high school players, $1394 \pm 66 \mathrm{~m}$ for the college players, and $1544 \pm 163 \mathrm{~m}$ for the WJBL players. After the movement distance per game of the players was analyzed by two-way factorial ANOVA for the factors win-loss ( 2 elements of win and loss) and grade group (3 elements of high school, college, and WJBL), only the main effect of the factor grade group was observed $(\mathrm{F}(1,24)=23.18, p<0.001)$. Thus, this main effect was tested by multiple comparison with Tukey's HSD test; as a result, significant differences were observed between the WJBL players and the high school and college players $(p<0.001)$. This means that the movement distance per game of the WJBL players was significantly longer than the high school and college players. However, because the analyzed time in each game differed, this finding does not conclude that the WJBL players, who were at higher competition levels, had more plentiful quantity of motion. The movement distances per game of the winning teams, although not statistically significantly, were longer than their opponent in all 3 games.

As a result of two-way factorial, repeated measure ANOVA for the factors grade group (3 elements) and game period ( 2 elements of 1 st and 2 nd halves), the main effect of the factor grade group $(\mathrm{F}(2,27)=25.27$, $p<0.001)$ and the interaction between the factors grade group and game period $(\mathrm{F}(2,27)=35.81, p<0.001)$ were significant. Thus, for this interaction, the simple main effects of the factor game period in the respective grade groups were tested. As a result, significant differences were observed in the simple main effects between the game periods in all grade groups $(\mathrm{F}(1,9)=40.37, p<0.001$ [high school]; 7.39, $p<0.05$ [college]; and 21.99, $p<0.001$ [WJBL]). Specifically, the movement distance per game of the college players was significantly longer in the 2nd half (3rd and 4th periods; $1410 \mathrm{~m}$ ) than the 1 st half (1st and 2 nd periods; $1378 \mathrm{~m}$ ) (i.e., $33 \mathrm{~m}$ longer) and that of the WJBL players was also significantly longer in the 2nd half $(1589 \mathrm{~m})$ than the 1 st half $(1499 \mathrm{~m})$ (i.e., $90 \mathrm{~m}$ longer), whereas that of the high school players was significantly shorter in the 2nd half $(1351 \mathrm{~m})$ than the 1 st half (1443 m) (i.e., $91 \mathrm{~m}$ shorter). Therefore, it was suggested that quantity of motion may need to be increased in the 2nd half depending on the game's momentum although quantity of motion is generally thought to be decreased in the 2 nd half compared to the 1st half (Matsumoto et al., 1998).

Further, the movement distances were analyzed by one-way factorial ANOVA for the factor playing position; where, point guard was abbreviated as PG, shooting guard was SG, forward was F, power forward was $\mathrm{PF}$, and center was $\mathrm{C}$. As a result, no significant main effects were observed. However, the mean movement distance of the PF position was the longest in the high school and college players $(5673 \mathrm{~m})$, whereas that of the $\mathrm{F}$ position was the longest in the WJBL players $(6393 \mathrm{~m})$. Conversely, the movement distance of the $\mathrm{C}$ position was the shortest in all positions of each grade group. The descending order of the high school players' movement distances was PF-FSG-PG-C, that of the high school players' movement distances was PF-SG-F-PG-C, and that of the WJBL players' movement distances was F-PG-SG-PF-C. The 
Table 1 Movement distances of the players andthe ball in high school game.

\begin{tabular}{cccccc}
\hline \multicolumn{1}{c}{ periods } & 1st & 2nd & 3rd & 4th & total \\
\hline ball & 2761 & 2981 & 2763 & 2754 & 11259 \\
A-High School & & & & & \\
PG & 1438 & 1490 & 1259 & 1296 & 5455 \\
SG & 1355 & 1496 & 1419 & 1302 & 5566 \\
F & 1483 & 1407 & 1459 & 1424 & 5681 \\
PF & 1501 & 1516 & 1341 & 1257 & 5433 \\
C & 1421 & 1414 & $1393 \pm 93$ & $1309 \pm 67$ & $5606 \pm 190$ \\
M S.D. & $1440 \pm 57$ & $1465 \pm 50$ & 1342 & 1422 & 5656 \\
B-High School & 1419 & 1473 & 1441 & 1277 & 5749 \\
PG & 1438 & 1456 & 1324 & 1332 & 5652 \\
SG & 1477 & 1413 & 1275 & 1279 & 5450 \\
F & 1381 & 1411 & $1365 \pm 74$ & $1337 \pm 63$ & 5335 \\
PF & 1370 & $1449 \pm 36$ & & $5568 \pm 170$ \\
M & $1417 \pm 43$ & & & & \\
\hline
\end{tabular}

Table 2 Movement distances of the players and the ball in college game.

\begin{tabular}{|c|c|c|c|c|c|}
\hline periods & $1 \mathrm{st}$ & 2 nd & $3 \mathrm{rd}$ & 4th & total \\
\hline ball & 2528 & 2677 & 2830 & 2676 & 10711 \\
\hline \multicolumn{6}{|l|}{ C-University } \\
\hline PG & 1389 & 1358 & 1466 & 1422 & 5635 \\
\hline SG & 1343 & 1427 & 1485 & 1403 & 5658 \\
\hline $\mathrm{F}$ & 1385 & 1379 & 1458 & 1318 & 5540 \\
\hline $\mathrm{PF}$ & 1452 & 1485 & 1529 & 1455 & 5921 \\
\hline C & 1297 & 1285 & 1372 & 1293 & 5247 \\
\hline $\mathrm{M} \pm \mathrm{S} . \mathrm{D}$ & $1373 \pm 58$ & $1387 \pm 75$ & $1462 \pm 57$ & $1378 \pm 69$ & $5600 \pm 243$ \\
\hline \multicolumn{6}{|l|}{ D-University } \\
\hline PG & 1296 & 1268 & 1350 & 1335 & 5249 \\
\hline SG & 1381 & 1462 & 1440 & 1343 & 5626 \\
\hline $\mathrm{F}$ & 1413 & 1330 & 1458 & 1470 & 5671 \\
\hline $\mathrm{PF}$ & 1373 & 1462 & 1481 & 1350 & 5666 \\
\hline $\mathrm{C}$ & 1373 & 1396 & 1435 & 1348 & 5552 \\
\hline $\mathrm{M} \pm \mathrm{S} . \mathrm{D}$ & $1367 \pm 43$ & $1384 \pm 85$ & $1433 \pm 50$ & $1369 \pm 57$ & $5553 \pm 176$ \\
\hline
\end{tabular}

Table 3 Movement distances of the players andthe ball in WJBL game.

\begin{tabular}{|c|c|c|c|c|c|}
\hline periods & $1 \mathrm{st}$ & $2 n d$ & $3 \mathrm{rd}$ & 4 th & total \\
\hline ball & 3141 & 2992 & 2797 & 3465 & 12395 \\
\hline \multicolumn{6}{|l|}{ E-team } \\
\hline PG & 1434 & 1438 & 1438 & 1792 & 6102 \\
\hline SG & 1598 & 1436 & 1400 & 1748 & 6182 \\
\hline $\mathrm{F}$ & 1598 & 1583 & 1581 & 1779 & 6541 \\
\hline $\mathrm{PF}$ & 1526 & 1467 & 1433 & 1720 & 6146 \\
\hline C & 1596 & 1499 & 1337 & 1750 & 6182 \\
\hline $\mathrm{M} \pm$ S.D. & $1550 \pm 72$ & $1485 \pm 61$ & $1438 \pm 90$ & $1758 \pm 28$ & $6231 \pm 177$ \\
\hline \multicolumn{6}{|l|}{ F-team } \\
\hline PG & 1601 & 1579 & 1433 & 2013 & 6626 \\
\hline SG & 1450 & 1412 & 1380 & 1833 & 6075 \\
\hline $\mathrm{F}$ & 1572 & 1438 & 1427 & 1807 & 6244 \\
\hline $\mathrm{PF}$ & 1473 & 1468 & 1364 & 1658 & 5963 \\
\hline C & 1411 & 1404 & 1240 & 1646 & 5701 \\
\hline $\mathrm{M} \pm$ S.D. & $1501 \pm 81$ & $1460 \pm 71$ & $1369 \pm 78$ & $1791 \pm 15$ & $6122 \pm 344$ \\
\hline
\end{tabular}


Table 4 Movement distances of the players and the ball for 1 minutes.

\begin{tabular}{|c|c|c|c|c|c|}
\hline periods & $1 \mathrm{st}$ & 2nd & $3 \mathrm{rd}$ & 4 th & Mean \\
\hline \multicolumn{6}{|l|}{ ball } \\
\hline High School & 189 & 177 & 193 & 193 & 188 \\
\hline college & 196 & 200 & 184 & 191 & 193 \\
\hline WJBL & 200 & 182 & 190 & 184 & 189 \\
\hline \multicolumn{6}{|l|}{ High School } \\
\hline A-team & $98.6 \pm 3.5$ & $87.2 \pm 3$ & $97.4 \pm 6.5$ & $91.6 \pm 4.7$ & $93.7 \pm 3.3$ \\
\hline B-team & $97.1 \pm 3.0$ & $86.5 \pm 2.2$ & $95.4 \pm 5.2$ & $93.5 \pm 4.4$ & $93.1 \pm 2.9$ \\
\hline \multicolumn{6}{|l|}{ College } \\
\hline C-team & $106.4 \pm 4.5$ & $103.5 \pm 5.6$ & $94.9 \pm 3.7$ & $98.4 \pm 5.0$ & $100.8 \pm 4.4$ \\
\hline D-team & $106.0 \pm 3.9$ & $103.2 \pm 7.3$ & $93 \pm 3.7$ & $97.8 \pm 4.6$ & $100.0 \pm 3.7$ \\
\hline \multicolumn{6}{|l|}{ WJBL } \\
\hline E-team & $96.8 \pm 4.6$ & $90.5 \pm 3.7$ & $97.8 \pm 6.1$ & $63.5 \pm 1.5$ & $95.2 \pm 2.8$ \\
\hline F-team & $95.6 \pm 4.7$ & $89.0 \pm 4.5$ & $93.1 \pm 2.3$ & $95.3 \pm 7.7$ & $93.3 \pm 4.3$ \\
\hline
\end{tabular}

behavioral characteristics of the playing positions are different among teams because roles of the playing positions are different depending on the team's collective tactics. In the present study, the high school and college teams were considered to tactically use plentiful quantity-of-motion players on SG, F, and PF rather than $\mathrm{PG}$ and $\mathrm{C}$ who play more often in the middle lane; the WJBL teams were considered to tactically employ plentiful quantity-of-motion players on outside playing positions like PG, SF, and F.

Moreover, the playing position with the longest movement distance in the team was $\mathrm{PF}$ for A-high school, SG for B-high school, PF for C-college, F for D-college, F for E-team, and PG for F-team. Among them, the PF of A (20 of 74 points), SG of B (15 of 68 points), $\mathrm{PF}$ of $\mathrm{C}$ ( 23 of 75 points), and $\mathrm{F}$ of $\mathrm{D}$ ( 21 of 68 points) were the team high scorers in the game.

Table 4 shows movement distances per minute of the players and the ball. As a result of three-way factorial, repeated measure ANOVA for the factors grade group (3 elements), playing position (5 elements), and game period ( 2 elements), the main effects of the factors grade group $(\mathrm{F}(2,15)=12.91, p<0.001)$ and game period $(\mathrm{F}(1,15)=10.45, p<0.01)$ and the interaction between the factors grade group and game period $(\mathrm{F}(2,15)=45.63, p<0.001)$ were significant. Thus, for this interaction, the simple main effects of the factor game period in the respective grade groups were tested. As a result, a significant difference was observed in the simple main effect of the factor game period only in the college players $(\mathrm{F}(1,9)=109.58, p<0.001)$. Unlike the movement distances per game, the movement distance per minute of the college players was significantly longer in the 1st half $(105 \mathrm{~m})$ than 2 nd half $(96 \mathrm{~m})$, whereas that of the high school players was not significantly different between the 1 st $(92 \mathrm{~m})$ and $2 \mathrm{nd}$ $(95 \mathrm{~m})$ halves and that of the WJBL players was also not significantly different between the 1 st $(94 \mathrm{~m})$ and 2nd (95 m) halves.

Further, the simple main effects of the factor grade group in the respective game periods (1st and 2 nd halves) were tested. As a result, a significant difference was observed only in the simple main effect of the factor grade group in the 1st half $(\mathrm{F}(2,27)=36.39$, $p<0.001)$. Thus, this main effect was tested by multiple comparison with Tukey's HSD test; as a result, significant differences were observed between the college players and the high school and WJBL players $(p<0.001)$. This means that the movement distance in the 1st half per minute of the college players was significantly longer than the high school and WJBL players.

\subsection{Maximum movement speed of players and ball}

Table 5 shows maximum movement speeds of the players and the ball in the high school, college, and WJBL games. The players with the fastest maximum movement speed among the high school, college, and WJBL players were SG in A $(7.82 \mathrm{~m} / \mathrm{s}), \mathrm{SG}$ in $\mathrm{D}$ $(7.80 \mathrm{~m} / \mathrm{s})$, and $\mathrm{SG}$ in $\mathrm{F}(8.98 \mathrm{~m} / \mathrm{s})$, respectively (i.e., all were $S G$ players).

After the maximum movement speed of the players was analyzed by two-way factorial ANOVA for the 
Table 5 The fastest movement speed of the players and the ball.

\begin{tabular}{|c|c|c|c|c|c|c|}
\hline \multirow{2}{*}{$\begin{array}{l}\text { Class } \\
\text { Team }\end{array}$} & \multicolumn{2}{|c|}{ High School } & \multicolumn{2}{|c|}{ College } & \multicolumn{2}{|c|}{ WJBL } \\
\hline & A-team & B-team & C-team & D-team & E-team & F-team \\
\hline ball & \multicolumn{2}{|c|}{15.62} & \multicolumn{2}{|c|}{15.52} & \multicolumn{2}{|c|}{16.79} \\
\hline PG & 7.60 & 7.20 & 7.23 & 7.59 & 8.07 & 8.02 \\
\hline SG & 7.82 & 7.32 & 7.53 & 7.80 & 8.45 & 8.98 \\
\hline $\mathrm{F}$ & 6.78 & 6.41 & 7.14 & 7.16 & 7.85 & 7.25 \\
\hline $\mathrm{PF}$ & 7.40 & 6.77 & 6.99 & 7.46 & 7.67 & 8.00 \\
\hline $\mathrm{C}$ & 6.68 & 6.31 & 7.59 & 7.56 & 8.01 & 7.81 \\
\hline $\mathrm{M} \pm$ S.D. & $7.27 \pm 0.49$ & $6.80 \pm 0.45$ & $7.29 \pm 0.26$ & $7.51 \pm 0.23$ & $8.01 \pm 0.29$ & $8.01 \pm 0.62$ \\
\hline
\end{tabular}

(Unit: $\mathrm{m} / \mathrm{s}$ )
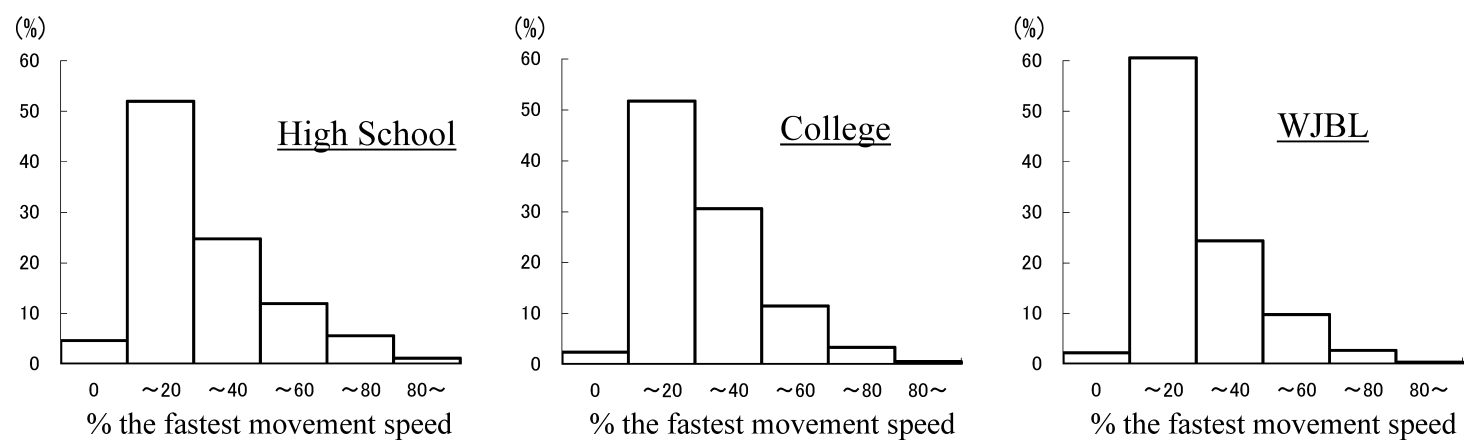

Figure 1 The distributiono of the fastest movement speed unde the game of high school, college, and WJBL player.

factors grade group (3 elements) and playing position (5 elements), the main effects of the factors grade group $(\mathrm{F}(2,15)=32.05, p<0.001)$ and playing position $(\mathrm{F}(4,15)=8.84, p<0.001)$ were significant. Thus, these main effects were each tested by multiple comparison with Tukey's HSD test; as a result, significant differences were observed in the main effect of the factor grade group between the WJBL players and the high school and college players $(p<0.001)$, indicating that the maximum movement speed of the WJBL players was significantly faster than that of the other players. Maximum movement speed is considered to be affected by individual tactics and individual capabilities such as running ability, probably not by tactical factors such as the team's collective tactics. The WJBL is the highest-level women's basketball league in Japan, composed of players who had achieved excellent performance as a high school and/or college player. The just-described findings may support this characteristic of the WJBL and suggest that the WJBL players had better individual capabilities.

On the other hand, significant differences were also observed in the main effect of the factor playing position between $\mathrm{SG}$ and $\mathrm{F}(p<0.001), \mathrm{SG}$ and $\mathrm{PF}$ $(p<0.05), \quad$ SG and $\mathrm{C}(p<0.01)$, and $\mathrm{PG}$ and $\mathrm{F}$ $(p<0.05)$. This means that the SG players exerted a maximum movement speed during the game that was significantly faster than the $\mathrm{F}, \mathrm{PF}$, and $\mathrm{C}$ players did, and that similarly, the PG players were significantly faster than the F players.

\subsection{Distribution of movement speed}

The total movement distance and maximum movement speed of the players alone were considered to be insufficient to provide effective knowledge about physical fitness factors during basketball games in order to present training instruction measures regarding physical fitness factors of players. Thus, the percentage of speed with respect to the maximum movement speed exerted by each player during the game (also called \% maximum movement speed) was calculated and was distributed in zones of $20 \%$ (e.g., a calculated percentage of $50 \%$ was distributed to the $41 \%-60 \%$ zone of the maximum movement speed). These frequency distributions of $\%$ maximum movement speed were used to investigate the movement speed during the game.

Figure 1 shows frequency distributions of \% maximum movement speed during the game of all 
players. In all grade groups, the appearance rate was the highest in the $1 \%-20 \%$ zone of the maximum movement speed (mean speed: $1.40 \mathrm{~m} / \mathrm{s}$ ) which corresponds to a jogging speed, and was the second highest in the $21 \%-40 \%$ zone of the maximum movement speed and the third highest in the 41\%-60\% zone of the maximum movement speed, indicating that higher movement speeds were less likely to appear. The appearance rates among grade groups, although slightly differing, were considered to be almost the same in distribution pattern. Specifically, the movement time at $\%$ maximum movement speeds of up to $20 \%$, whose movement intensity was relatively low, accounted for about $50 \%-55 \%$ of the length of time per game; that at $\%$ maximum movement speeds of $21 \%-60 \%$, whose intensity was middle, accounted for about $35 \%-40 \%$; and that at a $\%$ maximum movement speed of $61 \%$, whose intensity corresponds to almost full sprint, accounted for about 3\%-7\%; conversely, the standing time at a $\%$ maximum movement speed of $0 \%$ (i.e., neither walk nor run) accounted for about $2 \%-5 \%$ of the length of time per game.

\section{Discussion}

In basketball, players repeatedly dash, stop, and do a turn according to situations in games so as to run around on the court throughout the game. So, it is essential to objectively comprehend movement intensity and quantity of motion exerted by players during games for the devisal of training for performance enhancement. Thus, by targeting official basketball games, movement distances and speeds of players and the ball, previously-unrevealed factors, were quantitatively and objectively analyzed to obtain knowledge about physical fitness factors required for games and fundamental materials for training of those physical fitness factors.

\subsection{Movement distance}

The movement distance of the ball in the present study was $11,259 \mathrm{~m}$ in the high school game, $10,712 \mathrm{~m}$ in the college game, and $12,394 \mathrm{~m}$ in the WJBL game. The player having the longest movement distance in each grade group was PF in high A-school $(5,897 \mathrm{~m})$, $\mathrm{PF}$ in C-college $(5,921 \mathrm{~m})$, and $\mathrm{F}$ in F-team $(6,541 \mathrm{~m})$; the player having the shortest movement distance in each grade group was $\mathrm{C}$ in B-high school $(5,335 \mathrm{~m}), \mathrm{C}$ in C-college $(5,247 \mathrm{~m})$, and $\mathrm{C}$ in F-team $(5,701 \mathrm{~m})$.
These findings suggested: a reason why the PG and C players had shorter movement distances than the SG, F, and PF players may be that they played more often in the middle lane; and conversely, the SG, F, and PF players, who played often in the side lanes, may require plentiful quantity of motion because they need not only straight, back-and-forth movements on the court but also transverse movements between the sidelines such as side-changing. Therefore, because outside players who often move in the side lanes and between the sidelines on the front court perform obviously different quantity of motion from $\mathrm{C}$ players who often play in the middle lane, the former and latter players should be trained in a separate way. However, because the behavioral characteristics of the playing positions are different depending on the collective tactics employed by the team and capabilities of the team's players, further games should be analyzed to obtain more knowledge.

The playing position with the longest movement distance in each of the high school and college teams was that of the highest scorer of the game in her respective team. Because the present study analyzed only one game of each grade group these 4 highest scorers could not be compared to players in other games. However, on the basis that scores are related to the number of shots attempted and made, movements to shake marking players are required to increase the number of shots attempted, whereas excess movements may cause fatigue and decrease the percentage of shots made. Therefore, although whether movement distances directly affected the shots made was not revealed, it may be necessary to investigate the relationships of movement distances with team points scored and opponent points scored by studies with increased numbers of samples to be analyzed.

Further, movement distances per game of the winning teams were, although not statistically significantly, longer than their opponent in all 3 games of the present study. The number of offensive plays in the team was 80 in A-high school, 81 in B-high school, 80 in C-college, 78 in D-college, and 87 in E-team, and 88 in F-team. Higher numbers of offensive plays are presumed to lengthen the movement distances; in the present study, the number of offensive plays of Ccollege (the winning team) was higher than that of $\mathrm{D}$ college, whereas the winning teams of the high school and WJBL teams had lower numbers of offensive plays but longer movement distances. Winning or losing a game is affected by various factors, e.g., number of 
turnovers, percentage of shots made. The cause could not be discussed further because the present study analyzed only 3 games, and future studies are desired.

Moreover, for comparison of the movement distances of all grade groups, the movement distances per minute were analyzed. As a result, these movement distances of the high school players were not different from those of the WJBL players, and regarding the 1st half alone, those of the college players were significantly longer than those of the high school and WJBL players. The movement distances of the players in the present study were considered to represent their quantity of motion (Oba \& Okuda, 2007). It was suggested that content and proficiency of tactics, which differ in high school, college, and WJBL players, may be refined more in older grade groups, whereas quantity of motion, an index of game performance, was at equivalent levels among them.

\subsection{Movement speed}

Throughout the game, movement speeds of the players appeared over a broad range of 0 to about $8.0 \mathrm{~m} / \mathrm{s}$, and additionally, most of the speeds appearing were about $20 \%$ of the maximum movement speed of the players; these findings were fairly consistent with a previous study (Matsumoto et al., 1998). Specifically, the players moved at a speed corresponding to jogging during most of the game, whereas they ran at $60 \%$ (4.5 to $5.0 \mathrm{~m} / \mathrm{s}$ ) or more of the maximum movement speed the least during the game. Further, as shown in Figure 1 , the movement time at $\%$ maximum movement speeds of $21 \%-60 \%$ (about 2.0 to $4.5 \mathrm{~m} / \mathrm{s}$ ), which may exceed jogging speed, accounted for about $35 \%$ of the length of time per game (nearly 15 minutes), whereas the standing time at a $\%$ maximum movement speed of $0 \%$ accounted for about $2 \%-5 \%$ of the length of time per game (halftime, intervals between periods, and timeouts [about 1 to 2 minutes] were excluded from the analysis). Bishop and Wright (2006) indicated work rates during a basketball game of low: middle: high $=5: 4: 1$; the present study also indicated work rates of $1 \%$ $20 \%: 21 \%-60 \%: 61 \%-=5: 4: 1$, which may support the previous findings. In addition to these findings, it is obvious that under most situations, players move not at a constant speed but at a variety of speeds while changing their movement speed by acceleration, deceleration, quick direction change, etc (Oba \& Okuda, 2007). As pointed out by Matsumoto et al. (1998), and the findings of the present study, basketball players may require a high capability to exercise intermittently and that slow-fast training, e.g., interval training in which movements at top speeds and at relatively slow speeds are alternated, should be included in routine training. Moreover, moving at even a slow speed to recover stamina, instead of stopping fully, at rests during training may lead to further improvement in game performance.

The frequency distributions of percentage of movement speed had almost the same pattern among the grade groups. Similarly to the quantity of motion, therefore, the movement intensity did not differ among the grade groups, so movement intensity during practices may not need to be set based on grade groups. However, the maximum movement speeds were faster in the WJBL players than the high school and college players. The findings of the present study demonstrated: quantity of motion and work rates of the players during the game had similar patterns among the grade groups, whereas players' individual capabilities differed. The tactics employed by each team may be determined partly by individual capabilities of its players. Therefore, it may not be easy to employ tactics used in WJBL games for high school games, and it may be important to employ tactics in accordance with individual capabilities of players. At the same time, it may also be necessary to improve individual capabilities of players by training such as weight training and speed training.

In the present study, previously-unrevealed movements of players and the ball during basketball games were analyzed objectively and quantitatively. The objective analysis and quantification of movement distances and speeds of the players and the ball during actual games were considered to provide important materials for coaching, and the comparison among the grade groups and the playing positions may be significant because new knowledge was obtained. However, since playing positions in basketball change arbitrarily depending on offensive tactics unlike baseball, the present study, which fixed the playing position in the comparative analysis process, was considered to be insufficient to draw a firm conclusion. This is a limit of the present study. Moreover, since the number of games analyzed in the present study was one per grade group, the number of games to be analyzed should be increased for obtaining more validity and reliability. 


\section{References}

Bishop, D. C. \& Wright, C. (2006). A time-motion analysis of professional basketball to determine the relationship between three activity profiles: High, medium, and low intensity and the length of the time spent on court. International Journal of Performance Analysis in Sports, 6: 130-139.

Ishimura, U., Aoki, T. \& Naka, H. (1988). The influence and effect of Droughts on winning and losing in basketball: Universiade in Kobe. Bulletin of the Faculty of Education, Kanazawa University, Natural Science, 37: 119-124 (in Japanese with English abstract).

Iwamoto, Y., Aoki, T., Kadota, Y., Kato, T. \& Komura, K (1998). A cinematographic analysis of the one-on-one moves in basketball: The one-on-one moves in the give and go play. Bulletin of Tokyo Gakugei University, Section 5, Arts, Health and Sport Sciences, 50: 129-138 (in Japanese with English abstract).

Iwamoto, Y., Nakayama, D., Kadota, Y., Kato, T., Komura, K. \& Aoki, T. (2001) An analysis of two player techniques of basketball: On the pick and roll play. Bulletin of Tokyo Gakugei University, Section 5, Arts, Health and Sport Sciences, 53: 77-82 (in Japanese with English abstract).

Kan, A., Shiokawa, M., Okihara, K., Deguchi, T. \& Susa, T. (2000). Research on the fundamental data in game analysis of the Sanfrecce Hiroshima vs. Yokohama Marinos: Focus on the advantage of $3 \mathrm{D}$ and a computer. Medicine and Science of Soccer, 20: 19-24 (in Japanese).

Kato, H. (1999). The present condition and the subject of the tactics and computer analysis of soccer. Operations Research as a Management Science Research, 44: 125-131 (in Japanese).

Kato, T., Katsumoto, M. \& Irie, S. (1993). A study on offensive playing basketball games: Relation of two players position on setting up a passing play. Bulletin of the Faculty of Education, Ibaraki University (Educational Science), 42: 87-99 (in Japanese).

Kodama, Y., Suzuki, T. \& Yoshida, Y. (1996). Development of computer assisted tactics information system for basketball 2: Score database reference module. Bulletin of Sendai College, 27: 137-154 (in Japanese with English abstract).

Matsumoto, H., Wakayoshi, K. \& Ono, K. (1998). Methodologically comparative estimation for its demands between game and practice of college basketball club. The Japanese Journal of Sport Methodology, 11: 95-102 (in Japanese with English abstract).

Oba, W. \& Okuda, T. (2007). A study of movement distances and speed of the players and a ball in basketball game. The Japan Journal of Sport Methodology, 20: 71-84 (in Japanese with English abstract).

Oga, K., Hidaka, T., Uchiyama, H. \& Asai, K. (2001). A quantitative analysis of defensive ability in basketball. The Japanese Journal of Sport Methodology, 14: 41-49 (in Japanese with English abstract).

Ohashi, J. (1999). Game analysis of soccer: Application to the method and field. Japanese Journal of Biomechanics in Sports \& Exercise, 3: 119-124 (in Japanese).

Okuda, T., Oba, W. \& Doi, H. (2005). The present condition and the subject of game analysis research in basketball. Memories of Osaka Kyoiku University, Ser.IV (Education, Psychology, Special Education and Physical Education), 54: 203-212 (in Japanese with English abstract).

Okuda, T., Oba, W., Okihara, K. \& Kurokawa, T. (2007). A study on quantity analysis of the space in the basketball game: As a basic study of the group tactics. The Japan Journal of Sport Methodology, 20: 45-55 (in Japanese with English abstract).

Suzuki, J. (2005). The analysis of the basketball games by using the game reposts. Japan Journal of Sport Coaching, 4: 46-51 (in
Japanese with English abstract).

Suzuki, K. \& Nishijima, T. (2002). Causal structure of the attacking skill in soccer games. Japan Journal of Physical Education, Health and Sport Sciences, 47: 547-567 (in Japanese with English abstract).

Uchiyama, H. (2004). Structural analysis of team tactics in basketball. The Japan Journal of Sport Methodology, 17: 25-39 (in Japanese with English abstract).

Uchiyama, H. (2007). Elucidation of a method for study on sports tactics. Japan Journal of Physical Education, Health and Sport Sciences, 52: 133-147 (in Japanese with English abstract).

Uchiyama, H., Takei, M., Oga, K., Otaka, T. \& Sasaki, K. (2001). A case study on "transition" as collective tactics in basketball: Based on game analysis of the 18th Asian basketball championship for women. Bulletin of Sport Methodology: Institute of Health and Sport Science in University of Tsukuba, 24: 107-120 (in Japanese with English abstract).

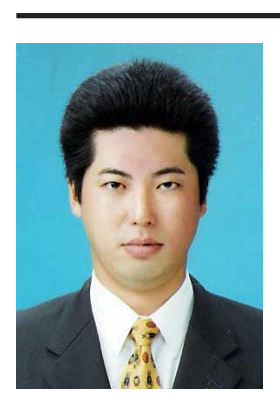

Name:

Wataru Oba

\section{Affiliation:}

Associate Professor, Faculty of Humanities, Okinawa University

\section{Address:}

555 Kokuba, Naha, Okinawa 902-8521

Brief Biographical History:

2000-2003 Doctoral Program, Graduate School of Education, Hiroshima University

2002-2004 Lecturer, Iwakuni Junior College

2003 Received PhD at Hiroshima University

2004-2007 Lecturer, Osaka Kyoiku University

2009- Associate Professor, Okinawa University

Main Works:

- Characteristics of prospective memory in Athletic Sports. Japanese Journal of Sport Psychology, 30(1): 9-19, 2003.

- A practical study of prospective memory on basketball games. The Japan Journal of Sport Methodology, 17: 1-8, 2004.

- A study of movement distances and speed of the players and a ball in basketball game. The Japan Journal of Sport Methodology, 20: 71-84, 2007.

- A study on analysis of the collective tactics action in basketball: Relationship to offensive success/failure and team area. The Japan Journal of Sport Methodology, 21(1): 47-50, 2007.

- A Study of Game Aspect and Game Performance in Elementary School Basketball Classes. The Bulletin of Japanese Curriculum Research and Development, 31(3): 29-38, 2008.

Membership in Learned Societies:

- Japan Society of Physical Education, Health and Sport Sciences

- Japanese Society of Sport Psychology

- The Japan Society of Sport Methodology

- The Japanese Psychological Association

- The Japanese Society for Cognitive Psychology

- Japan Curriculum Research and Development Association 